\title{
Identification of PLCL1 Gene for Hip Bone Size Variation in Females in a Genome-Wide Association Study
}

\author{
Yao-Zhong Liu ${ }^{1}$, Scott G. Wilson ${ }^{2,3}$, Liang Wang ${ }^{1,4}$, Xiao-Gang Liu ${ }^{1,4}$, Yan-Fang Guo ${ }^{1,4}$, Jian Li ${ }^{1}$, Han \\ Yan $^{1,4}$, Panos Deloukas ${ }^{3}$, Nicole Soranzo ${ }^{3,5}$, Usha Chinnapen-Horsley ${ }^{5}$, Alesandra Cervino ${ }^{5}$, Frances $\mathbf{M}$. \\ Williams $^{5}$, Dong-Hai Xiong ${ }^{6}$, Yin-Ping Zhang ${ }^{1,4}$, Tian-Bo Jin ${ }^{1,4}$, Shawn Levy ${ }^{7}$, Christopher J. Papasian ${ }^{1}$, \\ Betty M. Drees' ${ }^{1}$, James J. Hamilton ${ }^{1}$, Robert R. Recker ${ }^{6}$, Tim D. Spector ${ }^{3}$, Hong-Wen Deng ${ }^{1,4,6,8 *}$
}

1 School of Medicine, University of Missouri - Kansas City, Kansas City, Missouri, United States of America, $\mathbf{2}$ School of Medicine and Pharmacology, The University of Western Australia and Department of Endocrinology and Diabetes, Sir Charles Gairder Hospital, Nedlands, Western Australia, 3 Twin Research and Genetic Epidemiology Unit, St Thomas' Hospital, King's College London, London, United Kingdom, 4 The Key Laboratory of Biomedical Information Engineering of Ministry of Education and Institute of Molecular Genetics, School of Life Science and Technology, Xi'an Jiaotong University, Xi'an, People's Republic of China, $\mathbf{5}$ Wellcome Trust Sanger Institute, Wellcome Trust Genome Campus, Hinxton, United Kingdom, 6 Osteoporosis Research Center, Creighton University, Omaha, Nebraska, United States of America, 7 Vanderbilt Microarray Shared Resource, Vanderbilt University, Nashville, Tennessee, United States of America, 8 Laboratory of Molecular and Statistical Genetics, College of Life Sciences, Hunan Normal University, Changsha, Hunan, People's Republic of China

\begin{abstract}
Osteoporosis, the most prevalent metabolic bone disease among older people, increases risk for low trauma hip fractures (HF) that are associated with high morbidity and mortality. Hip bone size (BS) has been identified as one of the key measurable risk factors for HF. Although hip BS is highly genetically determined, genetic factors underlying the trait are still poorly defined. Here, we performed the first genome-wide association study (GWAS) of hip BS interrogating 380,000 SNPs on the Affymetrix platform in 1,000 homogeneous unrelated Caucasian subjects, including 501 females and 499 males. We identified a gene, PLCL1 (phospholipase c-like 1), that had four SNPs associated with hip BS at, or approaching, a genomewide significance level in our female subjects; the most significant SNP, rs7595412, achieved a $p$ value of $3.72 \times 10^{-7}$. The gene's importance to hip BS was replicated using the Illumina genotyping platform in an independent UK cohort containing 1,216 Caucasian females. Two SNPs of the PLCL1 gene, rs892515 and rs9789480, surrounded by the four SNPs identified in our GWAS, achieved $p$ values of $8.62 \times 10^{-3}$ and $2.44 \times 10^{-3}$, respectively, for association with hip BS. Imputation analyses on our GWAS and the UK samples further confirmed the replication signals; eight SNPs of the gene achieved combined imputed $p$ values $<10^{-5}$ in the two samples. The PLCL1 gene's relevance to HF was also observed in a Chinese sample containing 403 females, including 266 with HF and 177 control subjects. A SNP of the PLCL1 gene, rs3771362 that is only $\sim 0.6 \mathrm{~kb}$ apart from the most significant SNP detected in our GWAS (rs7595412), achieved a $p$ value of $7.66 \times 10^{-3}$ (odds ratio $=0.26$ ) for association with HF. Additional biological support for the role of PLCL1 in BS comes from previous demonstrations that the PLCL1 protein inhibits IP3 (inositol 1,4,5-trisphosphate)-mediated calcium signaling, an important pathway regulating mechanical sensing of bone cells. Our findings suggest that PLCL1 is a novel gene associated with variation in hip $\mathrm{BS}$, and provide new insights into the pathogenesis of $\mathrm{HF}$.
\end{abstract}

Citation: Liu Y-Z, Wilson SG, Wang L, Liu X-G, Guo Y-F, et al. (2008) Identification of PLCL1 Gene for Hip Bone Size Variation in Females in a Genome-Wide Association Study. PLoS ONE 3(9): e3160. doi:10.1371/journal.pone.0003160

Editor: Debbie Fox, The Research Institute for Children at Children's Hospital New Orleans, United States of America

Received June 25, 2008; Accepted August 18, 2008; Published September 8, 2008

Copyright: $\odot 2008$ Liu et al. This is an open-access article distributed under the terms of the Creative Commons Attribution License, which permits unrestricted use, distribution, and reproduction in any medium, provided the original author and source are credited.

Funding: Investigators of this work (Deng HW, Liu YZ, Wang L, Guo YF, Li J, Liu XG, Yan H, Xiong DH, Zhang YP, and Jing TB) were partially supported by grants from NIH (R01 AR050496-01, R21 AG027110, R01 AG026564, R21 AA015973 and P50 AR055081). The study also benefited from grants from National Science Foundation of China, Huo Ying Dong Education Foundation, HuNan Province, Xi'an Jiaotong University, and the Ministry of Education of China. Investigators from UK and Australia (Spector TD, Wilson SG, Deloukas P, Soranzo N, Chinnapen-Horsley U, Williams FM and Cervino A) were supported by Wellcome Trust; European Commission (QLK6-CT-2002-02629, GEFOS); the Arthritis Research Campaign; the Chronic Disease Research Foundation and the European Union FP-5 GenomEUtwin Project (QLG2-CT-2002-01254) and the Australian National Health and Medical Research Council (Project Grants 294402 and 343603 ).

Competing Interests: The authors have declared that no competing interests exist.

*E-mail: dengh@umkc.edu

\section{Introduction}

Osteoporosis is a serious public health problem in the elderly, leading to low trauma hip fractures $(\mathrm{HF})$ that are associated with high morbidity and mortality. Hip bone size (BS) is one of the major parameters for hip bone quality and strength, and it has been shown that abnormal hip BS contributes significantly to the pathogenesis of HF [1,2]. Consequently, hip BS has been identified as an important risk factor for HF.
Genetic factors play an important role in BS variation. One recent study demonstrated that the heritability of BS can reach as high as $75 \%$ [3], but specific genes underlying variation of BS, particularly hip BS, are still largely unknown. Only a limited number of association studies have been performed on hip BS to date, usually without full replication. They have implicated a few interesting candidate genes, such as VDR [4], COL1A2 [5] and CIP17 [6]. However each of these studies focused on genes with known significance in bone biology and, consequently, was not 
Table 1. Basic characteristics of study subjects.

\begin{tabular}{|c|c|c|c|c|c|}
\hline \multirow[t]{2}{*}{ Traits } & \multicolumn{2}{|c|}{ GWAS sample $(N=1,000)$} & \multirow[t]{2}{*}{$\begin{array}{l}\text { UK replication sample } \\
\text { (Female, } N=1,216 \text { ) }\end{array}$} & \multicolumn{2}{|c|}{ Chinese HF sample (Female, $N=403$ ) } \\
\hline & Female $(N=501)$ & Male $(N=499)$ & & Case $(N=226)$ & Control $(N=177)$ \\
\hline Age (years) & $50.1(17.7)$ & $50.5(18.9)$ & $48.7(11.8)$ & $72.1(8.9)$ & $67.7(6.3)$ \\
\hline Height (cm) & $163.8(6.5)$ & $177.8(7.0)$ & $162.1(6.2)$ & $157.8(4.7)$ & $153.2(6.7)$ \\
\hline Weight (kg) & $71.2(15.9)$ & $89.0(14.9)$ & $67.0(12.7)$ & $55.6(11.2)$ & $56.8(10.1)$ \\
\hline Hip BS $\left(\mathrm{cm}^{2}\right)$ & $34.2(3.4)$ & $44.7(4.5)$ & $33.6(3.9)$ & - & - \\
\hline
\end{tabular}

Note: Presented as means (SD)

doi:10.1371/journal.pone.0003160.t001

designed to identify potentially novel genes/regulatory mechanisms underlying hip BS.

A powerful strategy for identifying new genes associated with hip BS is genome-wide association studies (GWAS) that take advantage of the rapid development of high throughput SNP genotyping platforms combined with increasing knowledge of linkage equilibrium (LD) patterns in humans. The GWAS approach has demonstrated its great power to identify novel genes for human complex diseases/traits [7-11], using currently available high SNP density platforms that permit detection of culprit DNA changes within a narrow genomic region.

Here we conducted the first GWAS to search for novel genes underlying hip BS variation. Using Affymetrix $500 \mathrm{~K}$ arrays, we successfully genotyped and analyzed a total of $\sim 380,000$ SNPs in 1,000 unrelated Caucasians, including 501 females and 499 males. We identified a gene, PLCL1, that was associated with hip BS at the genome-wide significance level in female subjects. The gene's association with hip BS was replicated in an independent cohort from UK containing 1,216 Caucasian women. More importantly, the gene's relevance to $\mathrm{HF}$ was observed in a Chinese sample containing $266 \mathrm{HF}$ and 177 control subjects. (The subject characteristics of the cohorts used are detailed in Materials and Methods section and presented in Table 1.) Our findings strongly support the importance of the PLCL1 gene to hip BS, and to the pathogenesis of $\mathrm{HF}$.

\section{Results}

\section{GWAS findings}

We performed genome-wide genotypic association analyses for hip BS in our GWAS sample containing 1,000 subjects, but did not detect any SNP that passed the genome-wide significance threshold of $4.2 \times 10^{-7}$ in the total sample. We then proceeded with genderspecific association analyses and identified a SNP, rs7595412, that achieved a genome-wide significant $p$ value of $3.72 \times 10^{-7}$ in our female subjects. Rs7595412 is in intron 3 of the PLCL1 gene (Table 2 and Figure 1). Carriers of the major A allele of this SNP have on average $\sim 5 \mathrm{~cm}^{2}$ or $\sim 17 \%$ larger hip BS than non-carriers. In addition to the above SNP, three other SNPs in or near the PLCL1 gene (rs4850820, rs 10180112 and rs4850833) also achieved $p$ values approaching genome-wide significance $\left(p=4.61 \times 10^{-6}, 4.73 \times 10^{-6}\right.$ and $7.04 \times 10^{-6}$, respectively; Table 2). Of these three SNPs, two are in intron 5 and one is $\sim 72 \mathrm{~kb}$ downstream from the gene (Table 2 and Figure 1). Altogether these four SNPs span a region of $\sim 123 \mathrm{~kb}$, and are in strong LD (Figure 1). Additional details about these four SNPs are presented in Table 2 and Figure 1.

Table 2. Information for SNPs of the PLCL1 gene associated with hip BS or HF.

\begin{tabular}{|c|c|c|c|c|c|c|}
\hline SNP Name & Position & Role & Allele $^{1}$ & MAF $^{2}$ & MAF $^{3}$ & P Value/OR ${ }^{4}$ \\
\hline \multicolumn{7}{|c|}{ GWAS sample (association with hip BS) } \\
\hline rs7595412 & Chr2: 198670488 & Intron 3 & $A / G$ & 0.112 & 0.117 & $3.72 \times 10^{-7}$ \\
\hline rs4850820 & Chr2: 198690456 & Intron 5 & $\mathrm{G} / \mathrm{C}$ & 0.124 & 0.117 & $4.61 \times 10^{-6}$ \\
\hline rs10180112 & Chr2: 198691103 & Intron 5 & $\mathrm{G} / \mathrm{C}$ & 0.125 & 0.117 & $4.73 \times 10^{-6}$ \\
\hline rs4850833 & Chr2: 198793906 & downstream & $\mathrm{G} / \mathrm{A}$ & 0.139 & 0.125 & $7.04 \times 10^{-6}$ \\
\hline \multicolumn{7}{|c|}{ UK replication sample (association with hip BS) } \\
\hline rs892515 & Chr2: 198686066 & Intron 5 & $\mathrm{C} / \mathrm{T}$ & 0.309 & 0.350 & $8.62 \times 10^{-3}$ \\
\hline rs9789480 & Chr2: 198755871 & downstream & $\mathrm{C} / \mathrm{A}$ & 0.279 & 0.283 & $2.44 \times 10^{-3}$ \\
\hline \multicolumn{7}{|c|}{ Chinese HF sample (association with HF) } \\
\hline \multirow[t]{2}{*}{ rs3771362 } & Chr2:198671076 & Intron 3 & $\mathrm{C} / \mathrm{T}$ & 0.214 & 0.267 & $7.66 \times 10^{-3}$ \\
\hline & & & & & & $0.26(0.09-0.75)$ \\
\hline \multicolumn{7}{|c|}{$\begin{array}{l}\text { Note: } \\
{ }^{1} \text { The second allele represents the minor allele of each locus. } \\
{ }^{2} \text { Minor allele frequency calculated in our own Caucasian/Chinese sample. } \\
{ }^{3} \text { Minor allele frequency reported for Caucasians in the public database of HapMap CEU, except for the MAF of rs } 3771362 \text { that is from HapMap HCB for Chinese. } \\
{ }^{4} \text { Odds ratio with } 95 \% \text { confidence interval for the SNP, rs3771362, is for the minor allele. } \\
\text { doi:10.1371/journal.pone. } 0003160 . t 002\end{array}$} \\
\hline
\end{tabular}



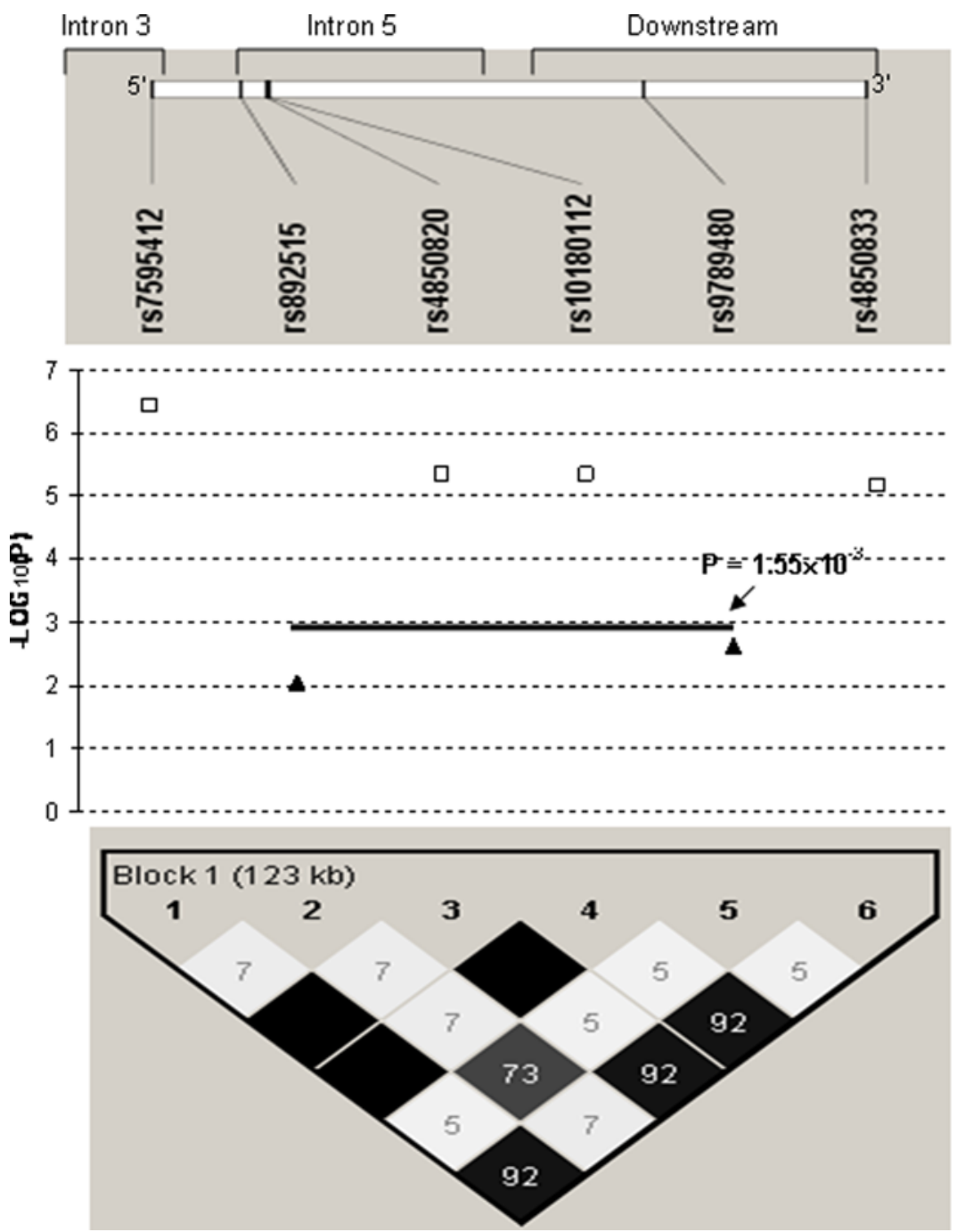

Figure 1. Association signals of PLCL1 gene in GWAS and UK replication samples. Note: 1. This haplotype block map was constructed using the most recent SNP genotype data (HapMap Data Rel 23a/phasell Mar 08, on NCBI B36 assembly, dbSNP b126) from HapMap (www.hapmap.org), showing pairwise LD in $r^{2}$. 2. The black bar represents the association signal $\left(p=1.55 \times 10^{-3}\right)$ achieved in the UK replication sample for the haplotype block formed by the two SNPs, rs892515 and rs9789480.

doi:10.1371/journal.pone.0003160.g001

To give a more comprehensive presentation of our GWAS findings, we present in Appendix S1 the most significant 30 SNPs detected genome-wide in the total sample as well as in each gender subgroup.

\section{Replication of the PLCL1 gene's association with BS in the UK sample}

In the UK replication sample, using a different genotyping platform, we also observed strong association signals for the PLCL1 gene with hip BS. We compared the association signals of our GWAS with those achieved in the GWAS performed in the UK cohort. The SNPs/genes that achieved $p$ values less than 0.01 in our GWAS were checked for $p$ values achieved in the UK cohort, and vice versa for those SNPs/genes that achieved $p$ values less than 0.01 in the UK cohort. As a result, the only gene that showed significant in silico replication signals is the PLCL1 gene that had four SNPs associated with hip BS in our GWAS $\left(p<10^{-5}\right)$ (Table 2) and two SNPs associated with hip BS in the UK cohort $(p<0.01)$ (Table 2).
A SNP in intron 5 of the PLCL1 gene, rs892515, achieved a $p$ value of $8.62 \times 10^{-3}$ for association with hip BS in the UK sample. That SNP is located between the most significant and the $2^{\text {nd }}$ most significant SNPs of the PLCL1 gene (i.e., rs7595412 and rs4850820, respectively) identified in our GWAS. The SNP rs892515 is separated from rs7595412 and rs4850820 by $\sim 15 \mathrm{~kb}$ and $\sim 4 \mathrm{~kb}$, respectively (Table 2 and Figure 1). Another SNP, rs9789480, located $\sim 35 \mathrm{~kb}$ downstream from the PLCL1 gene, achieved a $p$ value of $2.44 \times 10^{-3}$ in the UK sample. That SNP is positioned between two other interesting SNPs (i.e., rs 10180112 and rs4850833) of the PLCL1 gene identified in our GWAS ( $\sim 64 \mathrm{~kb}$ away from the former and $\sim 38 \mathrm{~kb}$ away from the latter SNP) (Table 2 and Figure 1). The haplotype formed by the two SNPs identified in the UK sample also achieved a $p$ value of $1.55 \times 10^{-3}$ for association with hip BS (Figure 1). Additional details about these two SNPs and their relative position in relation to the four SNPs identified in our GWAS is shown in Table 2 and Figure 1. As shown in Figure 1, rs892515 and rs9789480 have strong LD with each other, but weak LD with the four SNPs identified in our GWAS, even though these six SNPs are physically intermingled together. 
Imputation results on the region of the PLCL1 gene

As only $\sim 15 \%$ of SNPs overlap between the genotyping platform for our GWAS sample (i.e., Affymetrix) and that for the UK replication sample (i.e., Illumina), to better compare the association signals between the two samples, we imputed a common set of dense SNP markers covering the PLCL1 gene and its vicinity. The imputed association signals for the whole span of the gene are plotted in Figure 2. According to the imputation results, eight SNP markers achieved $p$ values less than $1 \times 10^{-6}$, and five additional SNP markers achieved $p$ values less than $1 \times 10^{-5}$ in our GWAS sample. In the UK replication sample, more than ten SNP markers achieved $p$ values less than 0.01 . More importantly, the strongest association signals for both samples overlap nicely in a region covering $\sim 3 / 4$ length of intron 5 and extending immediately downstream from the gene (shown in the Figure 2 as the region between the two dashed vertical lines).

Figure 3 zooms in on the region between the two dashed lines, highlighted in Figure 2, to examine this region more closely; only those SNPs that achieved $p$ values less than $1 \times 10^{-5}$ in the GWAS sample, and those that achieved $p$ values less than 0.05 in the UK sample, are shown. Detailed information for some of the plotted SNPs is presented in Table 3. A noticeable region in intron 5 with strong replication signals is highlighted in Figure 3 within the dashed rectangle (also designated "Region I" in Table 3). That region is less than $6 \mathrm{~kb}$ and contains nine significant SNP markers, according to imputation results. In that small region, two SNPs achieved genome-wide significant $p$ values $\left(p<4.2 \times 10^{-7}\right)$ and two achieved $p$ values less than $1 \times 10^{-5}$ in the GWAS sample; four other SNPs in this region achieved $p$ values less than 0.01 in the UK sample. Another interesting region in intron 5 (demarcated in Figure 3 as inside brackets, and designated "Region II" in Table 3) is flanked by two SNPs, rs989056 and rs10168722, both of which achieved genome-wide significant $p$ values $\left(p<4.2 \times 10^{-7}\right)$ in the GWAS sample. In the UK sample, this region of $\sim 11 \mathrm{~kb}$ contained three SNPs with $p$ values less than 0.01 and two additional SNPs with $p$ values less than 0.05.
Using Fisher's method [12], we combined the imputed $p$ values achieved in the two samples. We found that eight SNPs of the PLCL1 gene achieved combined imputed $p$ values less than $10^{-5}$, another five SNPs achieved combined $p$ values less than $10^{-4}$, and fifteen more SNPs achieved combined $p$ values less than 0.01 . These SNPs are mainly those as shown in Figure 3. Additional details of these SNPs are presented in Table 4.

\section{PLCL1 gene's importance to HF}

To further examine the PLCLI gene's importance to HF, we took advantage of a recently completed GWAS of HF in Chinese (containing 403 females and 297 males). We re-analyzed SNPs of the PLCL1 gene, used for the previous GWAS, for association with $\mathrm{HF}$ in Chinese female subjects. The SNP that achieved the most significant $p$ value in our GWAS in Caucasians (rs7595412) is not polymorphic in Chinese. However, a neighboring SNP, rs3771362, that is only $\sim 0.6 \mathrm{~kb}$ from $\mathrm{rs} 7595412$ achieved a $p$ value of $7.66 \times 10^{-3}$ for association with $\mathrm{HF}$ in the Chinese females, with an odds ratio (95\% CI) of $0.26(0.09-0.75)$ (Table 2). The SNP was not found to be associated with $\mathrm{HF}$ in the male subjects of the Chinese sample.

\section{PLCL1 gene's importance to other relevant phenotypes}

In the female subjects of our GWAS cohort, we also analyzed the PLCL1 gene's importance to other phenotypes relevant to osteoporosis, including hip and spine BMD, spine BS and height. The SNPs under analysis were the four SNPs of the gene that achieved the most significant results for association with hip BS in the female subjects of our GWAS cohort (Table 2).

We did not find significant association of the SNPs with hip and spine BMD and with height. However, we found marginally significant association of the SNPs with spine BS. The $p$ values achieved by the SNPs (rs7595412, rs4850820, rs 10180112 and rs4850833) are 0.048, 0.092, 0.092, and 0.056, respectively.

\section{OGWAS Sample A Replication Sample}

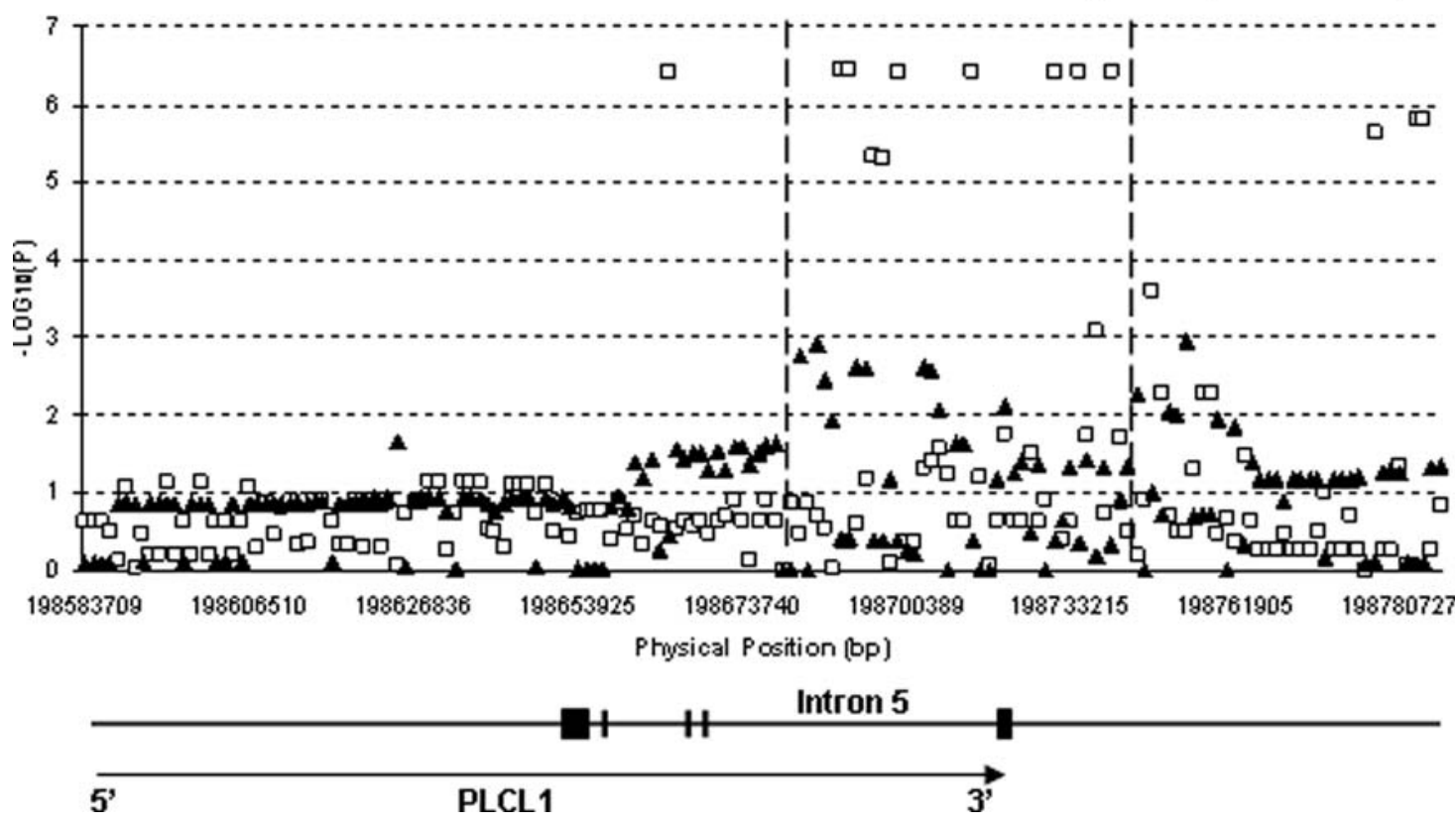

Figure 2. Imputation results for the PLCL1 gene in our GWAS and UK replication samples. doi:10.1371/journal.pone.0003160.g002 
Table 3. Major imputation results for intron 5 of the PLCL1 gene.

\begin{tabular}{|c|c|c|c|c|c|c|}
\hline \multirow[t]{2}{*}{ Region } & \multicolumn{3}{|c|}{ GWAS Sample } & \multicolumn{3}{|c|}{ Replication Sample } \\
\hline & SNP & Position (kb) & $P$ value & SNP & Position (kb) & $P$ value \\
\hline \multirow[t]{5}{*}{ I } & rs1808850 & 10.0 & $3.49 \times 10^{-7}$ & rs3771365 & 8.6 & $1.23 \times 10^{-3}$ \\
\hline & rs1371661 & 10.7 & $3.67 \times 10^{-7}$ & rs892515 & 9.2 & $3.45 \times 10^{-3}$ \\
\hline & rs4850820 & 13.6 & $4.61 \times 10^{-6}$ & rs11681576 & 9.3 & $1.21 \times 10^{-2}$ \\
\hline & rs10180112 & 14.2 & $4.73 \times 10^{-6}$ & rs7603309 & 11.1 & $2.37 \times 10^{-3}$ \\
\hline & & & & rs17792650 & 12.4 & $2.47 \times 10^{-3}$ \\
\hline \multirow[t]{5}{*}{$\|$} & rs989056 & 22.6 & $3.83 \times 10^{-7}$ & rs11884188 & 27.6 & $2.45 \times 10^{-3}$ \\
\hline & rs10168722 & 33.9 & $3.77 \times 10^{-7}$ & rs3771367 & 28.4 & $2.61 \times 10^{-3}$ \\
\hline & & & & rs1821430 & 30.6 & $8.44 \times 10^{-3}$ \\
\hline & & & & rs3771368 & 32.3 & $2.32 \times 10^{-2}$ \\
\hline & & & & rs3771369 & 32.3 & $2.32 \times 10^{-2}$ \\
\hline
\end{tabular}

Note: Position $(\mathrm{kb})$ is for the location of a SNP inside intron 5 of the PLCL1 gene, calculated as the distance from the beginning of the intron. doi:10.1371/journal.pone.0003160.t003

BMI's effects on the PLCL1 gene's association with hip BS

Since osteoporosis incidence was found to be inversely associated with BMI [13], we also examined the influence of
BMI on the PLCL1 gene's association with hip BS. In our GWAS cohort (female subjects), BMI appears to have some effects on the PLCL1 gene's association with hip BS. Adjusting hip BS with BMI
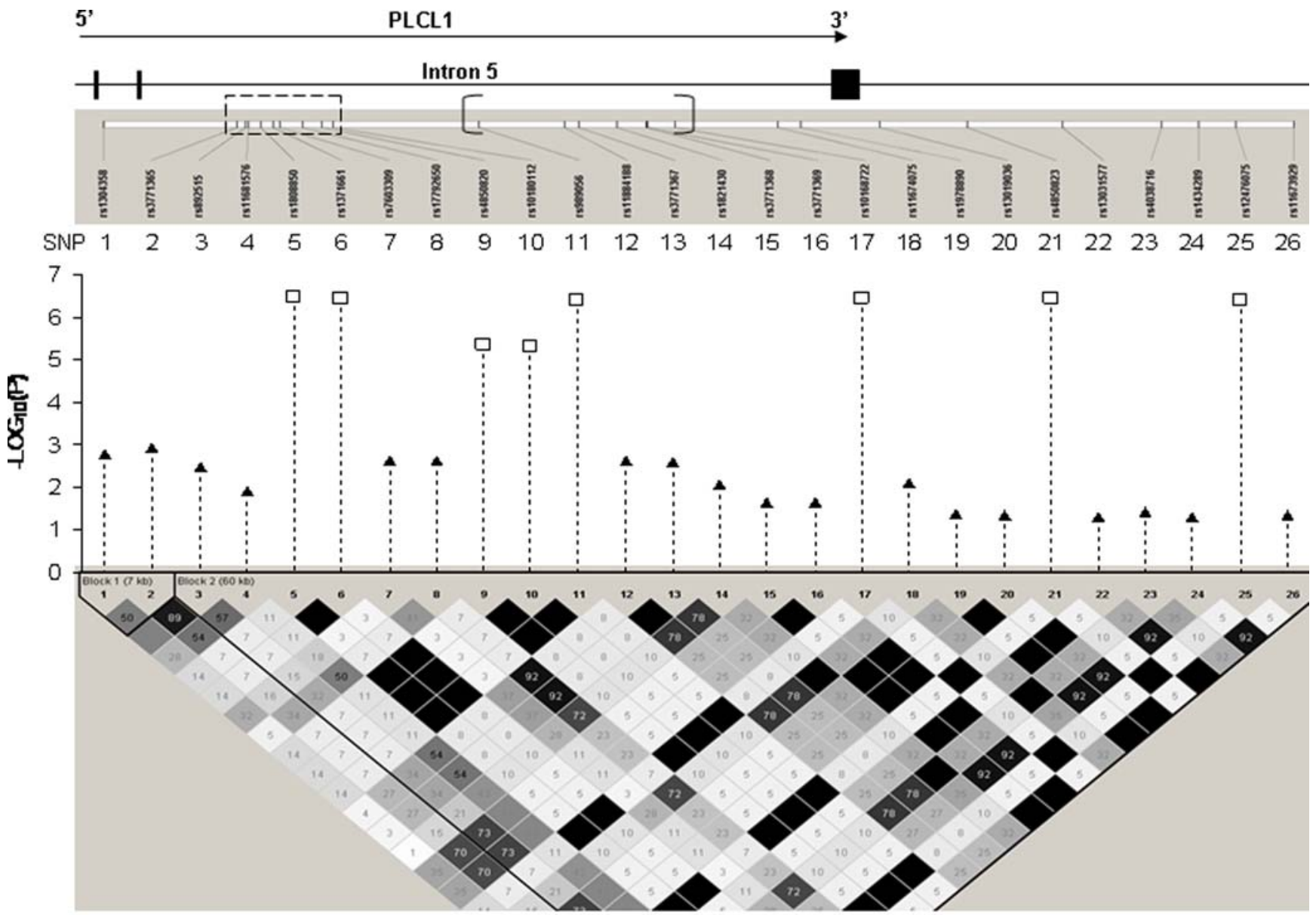

口GWAS Sample ^ Replication Sample

Figure 3. Association results imputed for intron 5 and the region immediately downstream from the $P L C L 1$ gene. doi:10.1371/journal.pone.0003160.g003 
Table 4. Combined imputed $p$ values for SNPs of the PLCL1 gene.

\begin{tabular}{|c|c|c|c|c|c|}
\hline SNP & Position & Role & $P$ value in GWAS & $P$ value in UK sample & Combined $\mathrm{P}$ \\
\hline rs7595412 & 198670488 & Intron 3 & $3.74 \times 10^{-7}$ & $3.53 \times 10^{-1}$ & $2.22 \times 10^{-6}$ \\
\hline rs1808850 & 198686906 & Intron 5 & $3.49 \times 10^{-7}$ & $3.95 \times 10^{-1}$ & $2.31 \times 10^{-6}$ \\
\hline rs1371661 & 198687636 & Intron 5 & $3.67 \times 10^{-7}$ & $3.97 \times 10^{-1}$ & $2.44 \times 10^{-6}$ \\
\hline rs 4850823 & 198727690 & downstream & $3.77 \times 10^{-7}$ & $4.10 \times 10^{-1}$ & $2.58 \times 10^{-6}$ \\
\hline rs10168722 & 198710808 & Intron 5 & $3.77 \times 10^{-7}$ & $4.12 \times 10^{-1}$ & $2.59 \times 10^{-6}$ \\
\hline rs989056 & 198699520 & Intron 5 & $3.83 \times 10^{-7}$ & $4.12 \times 10^{-1}$ & $2.63 \times 10^{-6}$ \\
\hline rs992603 & 198735269 & downstream & $3.77 \times 10^{-7}$ & $4.36 \times 10^{-1}$ & $2.73 \times 10^{-6}$ \\
\hline rs12476075 & 198743194 & downstream & $3.85 \times 10^{-7}$ & $4.53 \times 10^{-1}$ & $2.89 \times 10^{-6}$ \\
\hline rs4850447 & 198782135 & downstream & $1.51 \times 10^{-6}$ & $8.13 \times 10^{-1}$ & $1.80 \times 10^{-5}$ \\
\hline rs 2342753 & 198781579 & downstream & $1.58 \times 10^{-6}$ & $8.07 \times 10^{-1}$ & $1.85 \times 10^{-5}$ \\
\hline rs10183584 & 198778210 & downstream & $2.40 \times 10^{-6}$ & $7.50 \times 10^{-1}$ & $2.56 \times 10^{-5}$ \\
\hline rs4850820 & 198690456 & Intron 5 & $4.61 \times 10^{-6}$ & $4.06 \times 10^{-1}$ & $2.66 \times 10^{-5}$ \\
\hline rs10180112 & 198691103 & Intron 5 & $4.73 \times 10^{-6}$ & $4.06 \times 10^{-1}$ & $2.72 \times 10^{-5}$ \\
\hline rs3771367 & 198705284 & Intron 5 & $4.04 \times 10^{-2}$ & $2.61 \times 10^{-3}$ & $1.07 \times 10^{-3}$ \\
\hline rs11884188 & 198704457 & Intron 5 & $4.84 \times 10^{-2}$ & $2.45 \times 10^{-3}$ & $1.19 \times 10^{-3}$ \\
\hline rs11674075 & 198716751 & Intron 5 & $1.79 \times 10^{-2}$ & $7.77 \times 10^{-3}$ & $1.37 \times 10^{-3}$ \\
\hline rs 17792650 & 198689347 & Intron 5 & $6.78 \times 10^{-2}$ & $2.47 \times 10^{-3}$ & $1.62 \times 10^{-3}$ \\
\hline rs1821430 & 198707471 & Intron 5 & $2.69 \times 10^{-2}$ & $8.44 \times 10^{-3}$ & $2.14 \times 10^{-3}$ \\
\hline rs3771365 & 198685530 & Intron 5 & $1.89 \times 10^{-1}$ & $1.23 \times 10^{-3}$ & $2.17 \times 10^{-3}$ \\
\hline rs 9789480 & 198755871 & downstream & $3.14 \times 10^{-1}$ & $1.08 \times 10^{-3}$ & $3.06 \times 10^{-3}$ \\
\hline rs1434288 & 198740743 & downstream & $8.23 \times 10^{-4}$ & $6.30 \times 10^{-1}$ & $4.44 \times 10^{-3}$ \\
\hline rs 1304358 & 198677828 & Intron 5 & $3.29 \times 10^{-1}$ & $1.70 \times 10^{-3}$ & $4.75 \times 10^{-3}$ \\
\hline rs7603309 & 198688008 & Intron 5 & $2.50 \times 10^{-1}$ & $2.37 \times 10^{-3}$ & $5.00 \times 10^{-3}$ \\
\hline rs 4038716 & 198738934 & downstream & $1.88 \times 10^{-2}$ & $3.88 \times 10^{-2}$ & $5.99 \times 10^{-3}$ \\
\hline rs892515 & 198686066 & Intron 5 & $2.80 \times 10^{-1}$ & $3.45 \times 10^{-3}$ & $7.68 \times 10^{-3}$ \\
\hline rs1119850 & 198751505 & downstream & $5.25 \times 10^{-3}$ & $1.88 \times 10^{-1}$ & $7.80 \times 10^{-3}$ \\
\hline rs6434957 & 198757353 & downstream & $5.21 \times 10^{-3}$ & $1.92 \times 10^{-1}$ & $7.91 \times 10^{-3}$ \\
\hline rs6735486 & 198757463 & downstream & $5.21 \times 10^{-3}$ & $1.94 \times 10^{-1}$ & $7.96 \times 10^{-3}$ \\
\hline
\end{tabular}

in our GWAS sample increased the $p$ values for the PLCL1 gene's association with hip BS as shown in Table 2. After the adjustment, the $p$ values for the SNPs rs7595412, rs4850820, rs 10180112, and rs4850833 were increased to $1.15 \times 10^{-4}, 4.85 \times 10^{-4}, 4.85 \times 10^{-4}$, and $3.03 \times 10^{-4}$, respectively, as compared to the $p$ values before the adjustment, which were $3.72 \times 10^{-7}, 4.61 \times 10^{-6}, 4.73 \times 10^{-6}$, and $7.04 \times 10^{-6}$, respectively. The results suggest that the PLCL1 gene's association with hip BS may be partially mediated by BMI.

\section{Analyses for potential population stratification}

To detect potential stratification of our GWAS sample, we analyzed our sample using software Structure 2.2 [14]. When 2,000 randomly selected un-linked markers were used to cluster our subjects, under all assigned values (i.e., 2, 3, or 4) for the assumed number of population strata, $k$, all subjects of the sample were tightly clustered together, suggesting no population stratification. The results are shown in Appendix S2.

We further tested our GWAS sample for population stratification using the genomic control method [15]. Based on genomewide SNP information, we estimated the inflation factor $(\lambda)$, a measure for population stratification. Ideally, for a homogeneous population with no stratification, the value of $\lambda$ should be equal or near to 1.0. In our sample, the estimated $\lambda$ value was 1.007 , suggesting essentially no population stratification and further confirming the results achieved through the Structure 2.2 software.

We also analyzed the UK replication and Chinese HF samples for population stratification using the same approach described above for our GWAS sample, and achieved similar results. For the UK cohort, the subjects under study were those that remained after we excluded subjects who were not of European ancestry, according to analyses with Structure 2.2 [14]. The $\lambda$ value for the UK sample was 1.02 according to the genomic control analysis [15]. For the Chinese HF sample, the Structure program showed that all subjects were clustered together as a homogeneous population, and a $\lambda$ value of 1.02 was achieved through analysis with the genomic control method [15].

\section{Other analyses}

Using the Q-Q plot, we examined the distribution of $p$ values achieved in our GWAS for all of the $\sim 380,000$ SNPs that were analyzed (Appendix S3). As shown in the plot, the observed $p$ values match reasonably well with the expected $p$ values over a wide range of values of $\left[-\operatorname{LOG}_{10}(p)\right]$, which is from 0 to $\sim 4$. Observed $p$ values gradually depart from expected $p$ values at the extreme tail, where $\left[-\mathrm{LOG}_{10}(p)\right]$ is $\geq \sim 4$. The pattern suggests that our GWAS association findings were more likely due to true genetic variation than potential bias, such as genotyping errors. 
Using the FASTSNP program [16], we analyzed the potential functions for the identified SNPs as shown in Table 2. According to the analyses, three SNPs identified in our GWAS sample, rs7959412, rs4850820 and rs10180112, and a SNP identified in the UK sample, rs892515, are potential intronic enhancers. A polymorphic $\mathrm{A} \rightarrow \mathrm{G}$ change at rs7959412 and a polymorphic $\mathrm{G} \rightarrow \mathrm{C}$ change at rs4850820 and rs10180112 may eliminate binding sites for the transcription factors (TFs), GATA-1 and $\mathrm{CdxA}$, respectively, whereas a polymorphic $\mathrm{C} \rightarrow \mathrm{T}$ change at rs892515 may create a binding site for TFs, GATA-1 and XFD-1.

We compared the results of our GWAS with that of our most recently performed whole genome linkage study (WGLS) of BS [17]. In our previous WGLS the most significant region linked to hip BS was 8q24 that achieved a LOD score of 3.27 in the total sample and a LOD score of 3.01 in the female subgroup. Several genes in that region also achieved very strong association signals in our current GWAS. Two SNPs of the ZFAT1 (zinc finger gene in autoimmune thyroid disease 1) gene, rs7462652 and rs7006328, achieved $p$ values of $3.71 \times 10^{-5}$ and $5.03 \times 10^{-5}$, respectively, for association with hip $\mathrm{BS}$ in the total sample in our GWAS. In addition, three genes, DEPDC6 (DEP domain containing 6), EIF2C2 (eukaryotic translation initiation factor 2c, subunit 2) and COLEC10 (collectin 10), each had a SNP (rs9297608, rs2977451 and rs 16892015, respectively) associated with hip BS in the female subjects in our GWAS, with $p$ values of $5.42 \times 10^{-5}, 5.86 \times 10^{-5}$ and $1.05 \times 10^{-4}$, respectively.

\section{Discussion}

This study represents the first published GWAS for hip BS, an important risk factor for HF. Through this study we identified a novel gene, PLCL1 that had four SNPs associated with hip BS at, or approaching, the genome-wide significance level in our female subjects. The gene's association with hip BS was replicated in an independent UK sample despite it using a different platform and selection of SNPs. Two SNPs in the UK sample, that are surrounded by the four interesting SNPs identified in our GWAS, were associated with hip BS with $p$ values less than 0.01 (Figure 1). Imputation analyses of our GWAS and the UK samples showed a significant overlap of association signals in intron 5 of the PLCL1 gene and immediately downstream from that gene (Figure 2). In particular, according to the imputation analyses, a narrow window of $<6 \mathrm{~kb}$ in intron 5 contained four SNPs that achieved $p$ values less than $1 \times 10^{-5}$ (including two of genome-wide significance) in our GWAS sample, and four SNPs that achieved $p$ values less than 0.01 in the UK sample, (Table 3 and Figure 3). Combining $p$ values of the imputed SNPs in our GWAS and the UK samples, we found that eight SNPs of the PLCL1 gene achieved combined $p$ values less than $10^{-5}$, five additional SNPs achieved combined $p$ values less than $10^{-4}$, and fifteen more SNPs achieved combined $p$ values less than 0.01 (Table 4 ).

Importantly, we also demonstrated the relevance of PLCL1 to HF. A SNP of this gene, rs 3771362 , that is only $\sim 0.6 \mathrm{~kb}$ away from the most significant SNP identified in our GWAS (rs7595412), achieved a $p$ value of $7.66 \times 10^{-3}$ and an odds ratio of $0.26(95 \% \mathrm{CI}$ : 0.09-0.75) for association with $\mathrm{HF}$ in an independent Chinese sample. PLCL1 encodes an inositol 1,4,5-trisphosphate (IP3) binding protein that can inhibit IP3 mediated calcium signaling [18], an important pathway that regulates the response of bone cells to mechanical signals [19,20]. Overall, our association findings, together with PLCL1's potential functional relevance to bone mechanical sensing, provide strong evidence for the gene's importance for hip BS variation and the pathogenesis of $\mathrm{HF}$.

In our GWAS sample, the PLCL1 gene's association with hip BS was limited only to women, suggesting female-specificity of this gene's importance to osteoporosis. The female-specificity of the gene cannot be determined in the UK replication cohort since the cohort contains only women but no men [21]. However, analysis results in the Chinese HF cohort seem to support the femalespecificity of the gene as the gene was associated with $\mathrm{HF}$ in females but not in males (data not shown). Our findings are consistent with previous studies suggesting that genetic control of bone parameters may be gender-specific [22-24].

In this study, replication of the PLCL1 gene's association with hip BS in the UK sample was achieved "gene-wise" rather than "SNP-wise". The most significant SNPs of the gene detected/ imputed in our GWAS sample did not achieve nominally significant $p$ values in the UK sample (Table 4), although the use of different genotyping platforms may be partly responsible. However, significant replication signals did show up in the UK sample in many other SNPs of the PLCL1 gene, which are in neighborhood of those significant SNPs found in the GWAS sample, according to both the experimental data (Table 2 and Figure 1) and the imputation data (Table 3 and Figures $2 \& 3$ ).

BS can be measured as bone volume $\left(\mathrm{cm}^{3}\right)$, bone area $\left(\mathrm{cm}^{2}\right)$, or bone length (or diameter), and each of these measurements is legitimate and reflects various properties of bone. Areal BS measured by DXA, as adopted in this study, is a useful and reliable bone phenotype. Compared with other types of BS measurements, areal BS is relatively precise and involves less exposure to radiation during measurement $[25,26]$, enhancing its safety and feasibility for large-scale clinical investigation. Areal BS is also highly correlated with bone strength [27] and osteoporotic fractures $[2,28,29]$, and this correlation is largely independent from BMD $[2,28,29]$. Consequently, genetic study of BS may provide a unique perspective to osteoporosis research, because current research is largely dominated by studies of BMD.

Population stratification and/or ethnic admixture can be an important source of spurious association in genetic association studies. These factors are unlikely to exist in our samples, however, and are therefore unlikely to interfere with our association results. The study cohort for our GWAS came from an apparently homogenous US mid-west white population, living in Omaha, Nebraska and its surrounding areas. The relative homogeneity of this population is largely due to the predominance of Caucasians as the major ethnic group in this area for many generations. According to the recent 2000 census, ethnic minorities made up only less than $3 \%$ of the entire population in the State of Nebraska. In addition, we found that the allelic frequencies of the significant SNPs in our GWAS sample are very similar to those reported in the typical and representative Caucasian samples used in the HapMap CEU (Table 2). Furthermore, using Structure 2.2 [14], we thoroughly analyzed study subjects used in our GWAS in order to detect potential sub-populations in the sample. In the analyses, all subjects tightly clustered together as a single group, suggesting no population substructure in our sample (Appendix S2). Calculated through the genomic control method [15], the measure for population stratification $(\lambda)$ for our GWAS sample, was 1.007, suggesting essentially no stratification. Using the same approaches, we analyzed the UK replication sample and our Chinese HF sample and achieved similar results. For the above reasons, the association results, as detected in our study, are not likely to be plagued by spurious associations due to population admixture/ stratification. Moreover the wide generalizability of the PLCL1 gene's association makes this unlikely

In summary, we identified a novel gene, PLCL1, associated with hip BS through a GWAS. Our success in replicating the association of this gene with hip BS in a UK sample and the relevance of this gene to $\mathrm{HF}$ in a Chinese cohort, combined with 
the gene's functional implication in mechanical sensing by bone cells, makes it a strong candidate for regulation of hip BS and a potential key factor in the pathogenesis of HF. Apparent female specificity for the gene's association with hip BS supports genderspecific genetic basis of osteoporosis as suggested by previous studies [23,24] and provides new insights into our understanding of differential HF risk between females and males. As a future direction of research, we will perform fine-mapping of the PLCL1 gene in an enlarged independent Caucasian cohort to validate the gene's importance to hip BS and HF, including replicating the two SNPs that showed association with hip BS in the UK cohort (Table 2). In addition, focused molecular functional studies will be pursued to clarify the mechanisms by which this gene regulates hip BS variation and contributes to HF risk. The gene may also be related to bone growth in general - which also merits further work.

\section{Materials and Methods}

\section{Subjects}

The study was approved by the required Institutional Review Boards of all involved Institutions. Signed informed-consent documents were obtained from all study participants before they entered the study.

For the GWAS, a random sample containing 1,000 unrelated participants, including 501 women and 499 men, was identified from our established and expanding genetic repertoire currently containing more than 6000 subjects. All of the subjects were US Caucasians of European origin. Individuals with chronic diseases and conditions that might potentially affect bone metabolism were excluded. These diseases/conditions included chronic disorders involving vital organs (heart, lung, liver, kidney, brain), serious metabolic diseases (diabetes, hypo- and hyper-parathyroidism, hyperthyroidism, etc.), other skeletal diseases (paget disease, osteogenesis imperfecta, rheumatoid arthritis, etc.), chronic use of drugs affecting bone metabolism (hormone replacement therapy, corticosteroid therapy, anti-convulsant drugs), malnutrition conditions (such as chronic diarrhea, chronic ulcerative colitis, etc.), etc. Subjects taking anti-boneresorptive or bone anabolic agents/drugs, such as bisphosphonates, were also excluded from this study. The purpose of the above exclusion criteria was to minimize the effects of environmental and therapeutic factors thought or known to influence the skeletal system and so improve power and ability to find genes.,

For our GWAS sample, areal BS values of total hip (proximal femur) were measured using dual energy X-ray absorptiometry (DXA) machines (Hologic Inc., Bedford, MA, USA) that were calibrated daily. The coefficients of variation $(\mathrm{CV})$ of the DXA measurements for BS were about $1.94 \%$. General relevant characteristics of the study subjects are presented in Table 1.

For replication of the PLCL1 gene's association with hip BS, an independent sample containing 1,216 women of Caucasian European ancestry from the UK were used. The sample was selected from the TwinsUK cohort (www.twinsuk.ac.uk), a population-based sample of Britons previously shown to be representative of singleton populations, and the United Kingdom population, in general [30]. The areal BS of total hip site (proximal femur) was measured by DXA machines (Hologic Inc, Bedford, MA) using standard protocols. Basic characteristics of the UK study subjects are presented in Table 1 .

To validate the relevance of the PLCL1 gene to HF, we used a Chinese sample containing 403 female subjects including 226 with low trauma osteoporotic HF and 177 controls. All subjects were unrelated northern Chinese Han adults living in the city of Xi'an and its neighboring areas. Affected individuals with low trauma $\mathrm{HF}$ were recruited from the affiliated hospitals and associated clinics of Xi'an Jiaotong University. Inclusion criteria for $\mathrm{HF}$ patients were (i) age $<80$ years and onset age $>55$ years (all subjects were postmenopausal); (ii) HF caused by minimal or no trauma, usually due to falls from standing height or less; (iii) fracture site at the femoral neck or inter-trochanter regions; (iv) $\mathrm{HF}$ was identified/confirmed through diagnosis of orthopedic surgeons/radiologists according to radiological reports and x-rays. Patients with pathological fractures (e.g. due to tumors) and highimpact fractures (e.g. due to motor vehicle accidents) were excluded. Control subjects were enrolled through local advertisements. They were geography- and age-matched to the patients. All controls were older than 55 years, postmenopausal, and had no fracture history. Additional exclusion criteria for the control subjects were the same as those for our GWAS sample. The basic characteristics of the Chinese study subjects are shown in Table 1.

\section{Genotyping}

GWAS sample. Genomic DNA was extracted from whole human blood using a commercial isolation kit (Gentra systems, Minneapolis, MN, USA) following protocols detailed in the kit. Genotyping with the Affymetrix Mapping $250 \mathrm{k}$ Nsp and Affymetrix Mapping $250 \mathrm{k}$ Sty arrays was performed in Vanderbilt Microarray Shared Resources (VMSR) (http://array.mc.vanderbilt.edu/) using the standard protocol recommended by Affymetrix. Genotyping calls were determined from the fluorescent intensities using the DM algorithm with a $0.33 P$-value setting [31] as well as the B-RLMM algorithm [32]. DM calls were used for quality control while the BRLMM calls were used for all subsequent data analysis. B-RLMM clustering was performed with 94 samples per cluster.

The final average BRLMM call rate across the entire sample reached the high level of $99.14 \%$. However, out of the initial fullset of 500,568 SNPs, we discarded 32,961 SNPs with sample call rates $<95 \%$, another 36,965 SNPs with allele frequencies deviating from Hardy-Weinberg equilibrium (HWE) $(p<0.001)$, and 51,323 SNPs with minor allele frequencies (MAF) $<1 \%$. Therefore, the final SNP set maintained in the subsequent analyses contained 379,319 SNPs, yielding an average marker spacing of $\sim 7.9 \mathrm{~kb}$ throughout the human genome.

UK replication sample. Subjects in the UK sample were genotyped with the Illumina Infinium assay across three genomewide SNP sets, using fully compatible SNP arrays (Hap300 Duo, Hap300 and Hap550). Strict quality control criteria were applied, resulting in exclusion of 2,605 SNPs for violating Hardy-Weinberg equilibrium $\left(p<10^{-4}\right), 8,866 \mathrm{SNPs}$ for low call rate $(<90 \%)$, and 710 SNPs for low MAF $(<0.01)$; 306,823 SNPs were retained for final association analyses.

Ghinese HF sample. Subjects in the Chinese sample were genotyped with the Affymetrix Human Mapping $500 \mathrm{~K}$ array set (Affymetrix, Santa Clara, CA) following the same protocol used for our GWAS sample.

Quality control procedures were as follows. First, only samples with a minimum of $95 \%$ call rate were included; the final mean BRLMM call rate of the entire sample reached a high level of $99.02 \%$. Second, out of the initial full set of 500,568 SNPs, we discarded: 1) SNPs with a call rate $<90 \%$ in both cases and controls $(n=54,845)$; 2) those deviating from Hardy-Weinberg equilibrium (HWE) in controls $(p<0.001, n=22,002)$; 3) those having a minor allele frequency $(\mathrm{MAF})<0.05$ in the total sample $(n=142,188)$. Therefore, $281,533 \mathrm{SNPs}$ were eventually available for subsequent association analyses.

\section{Statistical Analyses}

GWAS sample. Parameters including age, age ${ }^{2}$, height and weight were tested for their association with hip BS. Significant 
( $p \leq 0.05)$ terms were then included as covariates to adjust the raw BS values for subsequent analyses. The adjusted BS data, if not following normal distributions, were further subjected to BoxCox transformation into normal distribution. GWAS analyses were performed through genotypic association tests implemented in HelixTree 5.3.1 (Golden Helix, Bozeman, MT, USA). This commercial program incorporates the well known genetic analyses programs, FBAT and PBAT (http://www.biostat.harvard.edu/ $\sim$ fbat/default.html), and the associated statistical methods [33].

The genome-wide significance threshold was set at $p=4.2 \times 10^{-7}$, derived by Freimer and Sabatti [34] based on a gene-wise approach, and subsequently modified by Lencz et al. [35] taking into account a more accurate estimate of the total number of genes in the human genome.

UK replication sample. Raw hip BS values were adjusted through the same procedures, described above, for the GWAS sample. Association analyses were performed using the PLINK (QFAM) software package (version 1.01) (http://pngu.mgh. harvard.edu/purcell/plink/) accounting for family structure in the sample [36]. Some of the subjects are monozygotic twins, and for these sib-pairs, genotypic information for only one individual per pair was included in the analyses, since monozygotic twins share identical genetic information. Where a single dizygotic twin had missing data, or was excluded, the remaining sibling was treated as a singleton in the statistical analysis.

Chinese HF sample. Association analyses for the SNPs of the PLCL1 gene were performed using the PLINK software package (version 1.02) [36]. Odds ratio for the SNP rs3771362 was calculated using logistic regression as implemented in Minitab package (Minitab Inc., State College, PA).

Quality control analyses. To detect population stratification in our GWAS sample, which may lead to spurious association results, we used the software Structure 2.2 (http:// pritch.bsd.uchicago.edu/software.html) to investigate the potential substructure of our sample. The program uses a Markov chain Monte Carlo (MCMC) algorithm to cluster individuals into different cryptic sub-populations on the basis of multi-locus genotype data [14]. To ensure robustness of our results, we performed independent analyses under three assumed numbers for population strata $(k=2,3$, and 4), using 2,000 un-linked markers that were randomly selected across the entire genome. To confirm the results achieved through Structure 2.2, we further tested population stratification of our GWAS sample using a method of genomic control [15].

To investigate if the association findings in our GWAS were due to potential bias (e.g., genotyping error), we examined the distribution of $p$ values for all $\sim 380,000$ SNPs analyzed in our sample using the quantile-quantile (Q-Q) plot.

The program, Structure 2.2 [14], and the method of genomic control [15] were also applied to the UK replication sample and the Chinese HF sample to test for potential population stratification.

Other analyses. The SNPs of the PLCL1 gene genotyped in our GWAS sample were largely different from that genotyped in

\section{Reference List}

1. Seeman E, Duan Y, Fong C, Edmonds J (2001) Fracture site-specific deficits in bone size and volumetric density in men with spine or hip fractures. J Bone Miner Res 16: 120-127.

2. Deng HW, Xu FH, Davies KM, Heaney R, Recker RR (2002) Differences in bone mineral density, bone mineral content, and bone areal size in fracturing and non-fracturing women, and their interrelationships at the spine and hip. J Bone Miner Metab 20: 358-366.

3. Havill LM, Mahaney MC, Binkley L, Specker BL (2007) Effects of genes, sex, age, and activity on BMC, bone size, and areal and volumetric BMD. J Bone Miner Res 22: 737-746. the UK replication sample due to different genotyping platforms used for the two samples. In order to better compare association signals between the two samples at the region of this gene, we imputed a set of dense SNP markers common to both samples (HapMap data, NCBI Build 35) that cover the entire region based on the markers genotyped in the respective samples. To impute the genotypes of un-typed SNPs, we used the software IMPUTE [37] (http://www.stats.ox.ac.uk/ marchini/software/gwas/ impute.html). Based on the imputed genotypes, we performed single SNP association analyses using the software SNPTEST (http://www.stats.ox.ac.uk/ marchini/software/gwas/snptest. html) to impute $p$ values of un-typed SNPs. To quantify the overall significance of imputed association signals in both our GWAS and the UK samples, Fisher's method [12] was used to combine the individual $p$ values imputed in each of the two samples.

To explore potential functions of the interesting SNPs identified in our GWAS and the UK replication samples, we used the FASTSNP (function analysis and selection tool for SNPs) program (http://fastsnp.ibms.sinica.edu.tw) that analyzes SNP functions based on up-to-date information extracted from 11 external bioinformatic databases at query time [16].

\section{Supporting Information}

Appendix S1 The most significant 30 SNPs for hip BS detected in GWAS. Note: SNP A-1819962* does not have a dbSNP ID. The ID as shown in the table is an Affy ID.

Found at: doi:10.1371/journal.pone.0003160.s001 (0.11 MB DOC)

Appendix S2 Results of analyses of potential population stratification for the GWAS sample using Structure 2.2. Note: As shown is output of the software Structure 2.2, which clustered our study subjects using 2,000 randomly selected unlinked markers under three assumed numbers of population strata, $\mathrm{k}=2,3,4$.

Found at: doi:10.1371/journal.pone.0003160.s002 (0.04 MB TIF)

Appendix S3 Q-Q plots for the p values achieved in the GWAS Found at: doi:10.1371/journal.pone.0003160.s003 (0.05 MB TIF)

\section{Acknowledgments}

We thank Dr. Jianfeng Liu for his help in analyzing the data. We also thank the study participants and staff from the TwinsUK, staff of the DNA Collections and Genotyping Facilities at the Wellcome Trust Sanger Institute for sample preparation; Quality Control of the Twins UK cohort for genotyping (in particular Amy Chaney, Radhi Ravindrarajah, Douglas Simpkin, Cliff Hinds, and Thomas Dibling); Paul Martin and Simon Potter of the DNA and Genotyping Informatics teams for data handling.

\section{Author Contributions}

Conceived and designed the experiments: HWD. Performed the experiments: LW HY PD NS UCH AC FMW DX YZ TJ SEL. Analyzed the data: XL YG JL YP. Wrote the paper: YZL. Provided data for in-silico replication of GWAS: SGW. Contributed data for in silico replication: TDS. Helped revise the paper: TDS RR JPH BMD CP SGW.
4. Fang Y, van Meurs JB, Rivadeneira F, van Schoor NM, van Leeuwen JP, et al. (2007) Vitamin D receptor gene haplotype is associated with body height and bone size. J Clin Endocrinol Metab 92: 1491-1501.

5. Lei SF, Deng FY, Xiao SM, Chen XD, Deng HW (2005) Association and haplotype analyses of the COL1A2 and ER-alpha gene polymorphisms with bone size and height in Chinese. Bone 36: 533-541.

6. Zmuda JM, Cauley JA, Kuller LH, Ferrell RE (2001) A common promotor variant in the cytochrome P450c17alpha (CYP17) gene is associated with bioavailability testosterone levels and bone size in men. J Bone Miner Res 16: 911-917. 
7. Hunter DJ, Kraft P, Jacobs KB, Cox DG, Yeager M, et al. (2007) A genomewide association study identifies alleles in FGFR2 associated with risk of sporadic postmenopausal breast cancer. Nat Genet.

8. Easton DF, Pooley KA, Dunning AM, Pharoah PD, Thompson D, et al. (2007) Genome-wide association study identifies novel breast cancer susceptibility loci. Nature.

9. Rioux JD, Xavier RJ, Taylor KD, Silverberg MS, Goyette P, et al. (2007) Genome-wide association study identifies new susceptibility loci for Crohn disease and implicates autophagy in disease pathogenesis. Nat Genet 39: 596-604.

10. Duerr RH, Taylor KD, Brant SR, Rioux JD, Silverberg MS, et al. (2006) A genome-wide association study identifies IL23R as an inflammatory bowel disease gene. Science 314: 1461-1463.

11. Frayling TM, Timpson NJ, Weedon MN, Zeggini E, Freathy RM, et al. (2007) A common variant in the FTO gene is associated with body mass index and predisposes to childhood and adult obesity. Science 316: 889-894.

12. Fisher RA (1925) Statistical Methods for Research Workers. New York: Hafner.

13. Morin S, Tsang JF, Leslie WD (2008) Weight and body mass index predict bone mineral density and fractures in women aged 40 to 59 years. Osteoporos Int.

14. Pritchard JK, Stephens M, Donnelly P (2000) Inference of population structure using multilocus genotype data. Genetics 155: 945-959.

15. Devlin B, Roeder K (1999) Genomic control for association studies. Biometrics 55: 997-1004.

16. Yuan HY, Chiou JJ, Tseng WH, Liu CH, Liu CK, et al. (2006) FASTSNP: an always up-to-date and extendable service for SNP function analysis and prioritization. Nucleic Acids Res 34: W635-W641.

17. Shen H, Long JR, Xiong DH, Guo YF, Xiao P, et al. (2006) A genomewide scan for quantitative trait loci underlying areal bone size variation in 451 Caucasian families. J Med Genet 43: 873-880.

18. Lin X, Varnai P, Csordas G, Balla A, Nagai T, et al. (2005) Control of calcium signal propagation to the mitochondria by inositol 1,4,5-trisphosphate-binding proteins. J Biol Chem 280: 12820-12832.

19. Riddle RC, Taylor AF, Genetos DC, Donahue HJ (2006) MAP kinase and calcium signaling mediate fluid flow-induced human mesenchymal stem cell proliferation. Am J Physiol Cell Physiol 290: C776-C784.

20. Reich KM, Frangos JA (1991) Effect of flow on prostaglandin E2 and inositol trisphosphate levels in osteoblasts. Am J Physiol Cell Physiol 261: C428-C432.

21. Richards JB, Rivadeneira F, Inouye M, Pastinen TM, Soranzo N, et al. (2008) Bone mineral density, osteoporosis, and osteoporotic fractures: a genome-wide association study. Lancet 371: 1505-1512.

22. Xiong DH, Shen H, Xiao P, Guo YF, Long JR, et al. (2006) Genome-wide scan identified QTLs underlying femoral neck cross-sectional geometry that are novel studied risk factors of osteoporosis. J Bone Miner Res 21: 424-437.
23. Xiao P, Shen H, Guo YF, Xiong DH, Liu YZ, et al. (2006) Genomic regions identified for BMD in a large sample including epistatic interactions and genderspecific effects. J Bone Miner Res 21: 1536-1544.

24. Ioannidis JP, Ng MY, Sham PC, Zintzaras E, Lewis CM, et al. (2007) Metaanalysis of genome-wide scans provides evidence for sex- and site-specific regulation of bone mass. J Bone Miner Res 22: 173-183.

25. Kalender WA (1992) Effective dose values in bone mineral measurements by photon absorptiometry and computed tomography. Osteoporos Int 2: 82-87.

26. Genant HK, Engelke K, Fuerst T, Gluer CC, Grampp S, et al. (1996) Noninvasive assessment of bone mineral and structure: state of the art. J Bone Miner Res 11: 707-730.

27. Yang R, Wang S, Lin H, Liu T, Hang Y, et al. (1998) Differential effects of bone mineral content and bone area on vertebral strength in a swine model. Calcif Tissue Int 63: 86-90

28. Vega E, Ghiringhelli G, Mautalen C, Rey VG, Scaglia H, et al. (1998) Bone mineral density and bone size in men with primary osteoporosis and vertebral fractures. Calcif Tissue Int 62: 465-469.

29. Mazess RB, Barden H, Mautalen C, Vega E (1994) Normalization of spine densitometry. J Bone Miner Res 9: 541-548.

30. Andrew T, Hart DJ, Snieder H, de Lange M, Spector TD, et al. (2001) Are twins and singletons comparable? A study of disease-related and lifestyle characteristics in adult women. Twin Res 4: 464-477.

31. Di X, Matsuzaki H, Webster TA, Hubbell E, Liu G, et al. (2005) Dynamic model based algorithms for screening and genotyping over $100 \mathrm{~K}$ SNPs on oligonucleotide microarrays. Bioinformatics 21: 1958-1963.

32. Rabbee N, Speed TP (2006) A genotype calling algorithm for affymetrix SNP arrays. Bioinformatics 22: 7-12.

33. Lange C, DeMeo DL, Laird NM (2002) Power and design considerations for a general class of family-based association tests: quantitative traits. Am J Hum Genet 71: 1330-1341.

34. Freimer N, Sabatti C (2004) The use of pedigree, sib-pair and association studies of common diseases for genetic mapping and epidemiology. Nat Genet 36: 1045-1051.

35. Lencz T, Morgan TV, Athanasiou M, Dain B, Reed CR, et al. (2007) Converging evidence for a pseudoautosomal cytokine receptor gene locus in schizophrenia. Mol Psychiatry 12: 572-580.

36. Purcell S, Neale B, Todd-Brown K, Thomas L, Ferreira MA, et al. (2007) PLINK: a tool set for whole-genome association and population-based linkage analyses. Am J Hum Genet 81: 559-575.

37. Marchini J, Howie B, Myers S, McVean G, Donnelly P (2007) A new multipoint method for genome-wide association studies by imputation of genotypes. Nat Genet 39: 906-913. 